\title{
Presence or absence of periodontal pathogens does not distinguish between chronic and aggressive periodontitis
}

\author{
Is it possible to distinguish between chronic and aggressive periodontitis based \\ on the presence of particular periodontal pathogens?
}

\begin{abstract}
Mombelli A, Casagni F, Madianos PN. Can presence or absence of periodontal pathogens distinguish between subjects with chronic and aggressive periodontitis? A systematic review. J Clin Periodontol 2002; 29(Suppl. 3):S10-S21.
\end{abstract}

Data Sources Data sources were Medline, with hand searches of the Journal of Dental Research, Journal of Clinical Periodontology, Journal of Periodontal Research and Journal of Periodontology dated from 1990 up to July 2001, and the reference lists of articles selected for inclusion.

Study selection Studies included were cross-sectional or longitudinal with microbiological data from at least two cohorts.

Data extraction and synthesis Information regarding quality and study characteristics was extracted independently by two reviewers. Kappa scores determined their agreement. Sensitivity and specificity of the microbiological tests were calculated for each selected study individually, with values being expressed as a Receiver Operator Characteristic (ROC) diagram.

Results The presence or absence of Actinobacillus actinomycetemcomitans could be evaluated in 11 papers; of Porphyromonas gingivalis in seven papers; and of Prevotella intermedia in six studies. Bactericides forsythus and Campylobacter rectus were each analysed in two papers. ROC diagrams indicated the limited discriminatory ability of all of the test parameters to identify subjects with aggressive periodontitis. An additional assessment showed that the highly leukotoxic variant of $A$. actinomycetemcomitans was uniquely associated with patients suffering from aggressive periodontitis. In a high proportion of patients diagnosed with the aggressive form of the condition, however, the presence of this variant could not be detected.

Conclusions The presence or absence of $A$. actinomycetemcomitans, $P$. gingivalis, $P$. intermedia, $B$. forsythus and $C$. rectus could not distinguish subjects with aggressive periodontitis from those with the chronic form.

\section{Commentary}

Using the diagnostic criteria of sensitivity and specificity, this systematic review tests the hypothesis that a single micro-organism can be used to differentiate between chronic and aggressive periodontal disease. The results demonstrate that, with the data currently available, the hypothesis is not true. The results are not surprising. Over the past 30 years it has become clear that periodontal disease is a mixed Gram-negative anaerobic infection. During the same period, it has also become clear that altered host responses alter the clinical manifestations of these infections.

Data from some 18 included studies are presented in the review. The authors acknowledge uncertainties within the clinical diagnoses found in the included studies. Nonetheless, the papers selected were worthy of review. Most of them had overlaps in the bacterial species being analysed. Sensitivity and specificity characteristics were considered for each study, using ROC diagrams.

Marsh $^{1}$ offers the unifying view that dental diseases are, "ecological catastrophes". The systematic review of adjunctive antibiotic use along with scaling and root planing ${ }^{2}$ supports this notion. The finding that antibiotic use consistently improves the clinical outcome of periodontal care (independent of infection type and antibiotic), suggests that antibiotics change the oral ecology from one consistent with disease to one more consistent with health. Thus, the notion of one-bacterium-one-diagnosis lacks support from multiple perspectives. Conversely, the results suggest that, for now, current clinical methods should remain in force, unless one is willing to employ adjunctive antibiotic therapy.

\section{Practice point}

- Based on current data the hypothesis that a single periodontal microorganism can differentiate between chronic and aggressive periodontal diseases remains unsubstantiated.

\section{Cataldo W. Leone}

Department of Periodontology and Oral Biology, Boston University Goldman School of Dental Medicine, Boston, Massachusetts, USA

1. Marsh PD. Are dental diseases examples of ecological catastrophes? Microbiology 2003; 149:279-294.

2. Herrera D, Sanz M, Jepsen S, Needleman I, Roldán S. A systematic review on the effect of systemic antimicrobials as an adjunct to scaling and root planing in periodontal patients. J Clin Periodontol 2002; 29(Suppl. 3):S136-S159.

Evidence-Based Dentistry (2003) 4, 60.

doi:10.1038/sj.ebd.6400204 